tendon and the plantar aponeurosis. Correlations between clinical, activity and US scores were investigated by SPPSS software.

Results: A total of 208 patients were included. At examination $88.9 \%$ had an active disease and $64.4 \%$ of SpA were taking NSAIDs. 6240 entheses were assessed clinically and with US. A positive correlation was found between activity scores and two clinical scores (Peripheral Enthesitis Score, SPARCC). A good correlation was found between Activity scores and all US scores on one hand and with SPARCC scores and PES scores on the other hand. For US and clinical correlations, only Doppler scores was positively correlated with 2 clinical scores (SPARCC and PES scores), Acute Enthesitis scores was correlated with the PES scores. No correlation was found between clinical scores and MASEI or SES scores. No correlation was found with the mean enthesitis VAS scores.

Table 1. Correlations between clinical scores and activity scores with ultrasound scores

\begin{tabular}{lccccc}
\hline scores & PES & VASm Enthestis & SPARCC & ASDAS-vs & ASDAS-crp \\
\hline Acute Enthesitis & $\mathrm{r}: 0,14$ & $\mathrm{r}:-0,03$ & $\mathrm{r}: 0,16$ & $\mathrm{r}: 0,43$ & $\mathrm{r}: 0,52$ \\
& $\mathbf{p}: \mathbf{0 , 0 4}$ & $\mathrm{p}: 0,72$ & $\mathrm{p}: 0,07$ & $\mathbf{p}<\mathbf{0 , 0 0 1}$ & $\mathbf{p}<0,001$ \\
Chronic Enthesitis & $\mathrm{r}: 0,07$ & $\mathrm{r}:-0,15$ & $\mathrm{r}: 0,06$ & $\mathrm{r}: 0,19$ & $\mathrm{r}: 0,22$ \\
& $\mathrm{p}: 0,30$ & $\mathrm{p}: 0,03$ & $\mathrm{p}: 0,41$ & $\mathbf{p}: \mathbf{0 , 0 0 6}$ & $\mathbf{p}: \mathbf{0 , 0 0 2}$ \\
Global Enthesitis & $\mathrm{r}: 0,15$ & $\mathrm{r}:-0,08$ & $\mathrm{r}: 0,13$ & $\mathrm{r}: 0,43$ & $\mathrm{r}: 0,52$ \\
& $\mathbf{p}: \mathbf{0 , 0 3}$ & $\mathrm{p}: 0,24$ & $\mathrm{p}: 0,07$ & $\mathbf{p}<\mathbf{0 , 0 0 1}$ & $\mathbf{p}<\mathbf{0 , 0 0 1}$ \\
Doppler Enthesitis & $\mathrm{r}: 0,16$ & $\mathrm{r}:-0,001$ & $\mathrm{r}: 0,18$ & $\mathrm{r}: 0,34$ & $\mathrm{r}: 0,36$ \\
& $\mathbf{p}: \mathbf{0 , 0 2}$ & $\mathrm{p}: 0,99$ & $\mathbf{p}: \mathbf{0 , 0 1}$ & $\mathbf{p}<\mathbf{0 , 0 0 1}$ & $\mathbf{p}<\mathbf{0 , 0 0 1}$ \\
MASEl & $\mathrm{r}: 0,09$ & $\mathrm{r}:-0,08$ & $\mathrm{r}: 0,12$ & $\mathrm{r}: 0,44$ & $\mathrm{r}: 0,34$ \\
& $\mathrm{p}: 0,19$ & $\mathrm{p}: 0,23$ & $\mathrm{p}: 0,07$ & $\mathbf{p}<\mathbf{0 , 0 0 1}$ & $\mathbf{p}<\mathbf{0 , 0 0 1}$ \\
SES & $\mathrm{r}: 0,04$ & $\mathrm{r}:-0,04$ & $\mathrm{r}: 0,09$ & $\mathrm{r}: 0,31$ & $\mathrm{r}: 0,38$ \\
& $\mathrm{p}: 0,55$ & $\mathrm{p}: 0,59$ & $\mathrm{p}: 0,18$ & $\mathbf{p}<\mathbf{0 , 0 0 1}$ & $\mathbf{p}<\mathbf{0 , 0 0 1}$ \\
\hline
\end{tabular}

Table 2. Correlations between clinical scores and activity scores

\begin{tabular}{lccc}
\hline & PES & VASm Enthestis & SPARCC \\
\hline ASDAS-vs & $r: 0,32$ & $r:-0,04$ & $r: 0,27$ \\
& $\mathbf{p}<0,001$ & $p: 0,62$ & $\mathbf{p}<0,001$ \\
ASDAS-crp & $r: 0,31$ & $r:-0,03$ & $r: 0,27$ \\
& $\mathbf{p}<\mathbf{0 , 0 0 1}$ & $\mathrm{p}: 0,62$ & $\mathbf{p}<\mathbf{0 , 0 0 1}$ \\
\hline
\end{tabular}

Conclusions: All US enthesitis scores were correlated with disease activity scores but those correlated with the clinical symptoms and not with its intensity were: Acute Enthesitis Scores, Global Enthesitis Scores and especially Doppler signal Enthesitis Scores.

Disclosure of Interest: None declared

DOI: 10.1136/annrheumdis-2017-eular.1968

\section{SAT0624 QUANTITATIVE 3D IMAGING OF TENOSYNOVITIS AND BONE MARROW EDEMA BY DCE-MRI IS A SENSITIVE MEASURE OF RESPONSE TO THERAPY IN RHEUMATOID ARTHRITIS}

C. Roberts $^{1}$, G. Guillard ${ }^{2}$, M.A. Bowes ${ }^{2}$, J. Burlison ${ }^{2}$, A. Khan ${ }^{2}$, N. Maguire ${ }^{1}$, S. Gotla ${ }^{1}$, A. Morgan ${ }^{1}$, G.J. Parker ${ }^{1,3}$, R. Hodgson ${ }^{3,4}$, J. Freeston ${ }^{4}$, E.M. Vital ${ }^{4}$ P. Bird ${ }^{5}$, P. Emery ${ }^{4}$, P.G. Conaghan ${ }^{4}$. ${ }^{1}$ Bioxydyn; ${ }^{2}$ Imorphics; ${ }^{3}$ Centre for Imaging Sciences, University of Manchester, Manchester; ${ }^{4}$ University of Leeds, Leeds, United Kingdom; ${ }^{5}$ Combined Rheumatology Practice, Sydney, Australia

Background: Quantitative analysis of tissue microvascular function using dynamic contrast-enhanced MRI (DCE-MRI) shows promise for improved understanding of synovial pathophysiology in rheumatoid arthritis (RA). Assessing tenosynovitis and bone marrow edema (BME) using physiologically-interpretable measures such as Ktrans may offer additional insights; this will require increased precision in quantification.

Objectives: To apply a 3D DCE-MRI method to quantify capillary permeability $\left(K^{\text {trans }}\right)$ in tendons, bone marrow edema, and to explore muscle involvement. To generate precise and consistent $3 \mathrm{D}$ regions of interest $(\mathrm{ROI})$ of tenosynovitis and BME using active appearance models (AAMs)? and evaluate response to therapy within these regions.

Methods: MR images of the hand were acquired in 27 patients with established RA who had recently commenced the same biological therapy. Subjects were imaged at $0,3,6$ months. The MRI protocol included pre- and post-contrast high-resolution 3D FLASH acquisitions. The DCE-MRI scan protocol included T1 mapping sequences followed by 15 sequential volumes acquired over 5 minutes, during which a bolus of gadolinium $(0.1 \mathrm{mmol} / \mathrm{kg})$ was administered at $2 \mathrm{ml} / \mathrm{s}$ at the beginning of the 5th volume measurement. The Extended Kety model was applied to each voxel concentration-time series within ROls, allowing voxelwise estimates of $K^{\text {trans }}$. Pre-contrast T1-weighted mages were searched using 3D active appearance models (AAMs) to reliably identify tendons and their sheath in the carpal tunnel region as well as bones and marrow area. Images from the dynamic series were registered to the high-resolution pre-contrast images, providing standardised $3 \mathrm{D}$ ROls for each of the regions. Median $K^{\text {trans }}$ was summarised in each patient for each ROI. T-test $(p<0.05)$ determined significant differences from baseline.

Results: Differences in baseline Ktrans were observed in each tissue type and demonstrated that highest levels of inflammation within the synovium, followed by tenosynovitis, BME and detection of low grade inflammation in the muscle (Fig. 1). Significant post-therapy responses in Ktrans, indicating a reduction in perfusion and capillary permeability associated with reduced inflammation, were seen in each of these tissue types (Fig.1). RAMRIS scoring showed no significant change at either 3 or 6 months. 3D visualisation of $K$ trans in tenosynovitis revealed additional spatial response to the biological treatment (Fig. 2) - most of the remaining tenosynovitis at 6 months had lower permeability with almost all of the higher permeability class of synovitis disappearing after therapy.

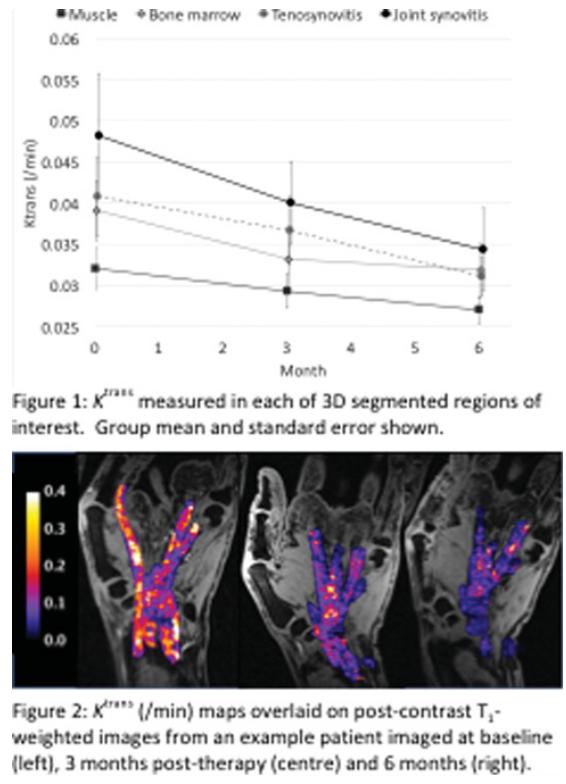

Conclusions: 3D DCE-MRI measures of tenosynovitis and bone marrow edema are practical in RA MR imaging trials, and offer sensitivity to change and differential tissue response not visible with other methods. Therapy-induced response in muscle suggests that there may be an inflammatory process in RA affecting local muscle groups.

Disclosure of Interest: None declared

DOI: 10.1136/annrheumdis-2017-eular.6584

\section{SAT0625 ULTRASOUND ASSESSMENT OF JOINTS AND ENTHESIS IN A COHORT OF PATIENTS AFFECTED BY OCHRONOSIS: HOW COMMON IS INFLAMMATION?}

C. Toscano ${ }^{1}$, V. Di Sabatino ${ }^{1}$, A. Adinolfi ${ }^{1}$, M. Bardelli ${ }^{1}$, V. Picerno ${ }^{1}$, E. Selvi ${ }^{1}$ A. Santucci $^{2}$, M. Galeazzi ${ }^{1}$, B. Frediani ${ }^{1}$, G. Filippou ${ }^{1} .{ }^{1}$ Department of Medicine, Surgery and Neuroscience, ${ }^{2}$ Department of Biotechnology, Chemistry and Pharmacy, University of Siena, Siena, Italy

Background: Ochronosis, the musculoskeletal manifestation of alcaptonuria $(A K U)$, is characterized by alterations of the spine and large joints of the limbs similar to those of osteoarthritis. However, some cases of spinal involvement that resembles spondiloarthritis $(\mathrm{SpA})$ have been describe, suggesting a prevalent inflammatory involvement of the joints.

Objectives: To evaluate the prevalence of inflammatory abnormalities in peripheral joints and enthesis of a cohort of patients affected by AKU.

Methods: consecutive patients with definite diagnosis of AKU (with or without clinical manifestations) referred at our clinic from 2014 to 2016 were enrolled. All patients underwent a US examination of the following sites bilaterally: metacarpophalangeal joints (MCP), proximal interphalangeal joints (PIP), radiocarpal/mid carpal joints, elbow, gleno-humeral, hip, knee, ankle and metatarso-phalangeal (MTP) joints; flexor and extensor tendons of fingers and wrist and the ankle tendons. Further, the enthesis of the rotator cuff of the shoulder, triceps, quadriceps, patellar and Achilles tendon were assessed. Joints and tendons with a synovial sheath were assessed for effusion, synovial hypertrophy and power Doppler (PD) signal while enthesis were evaluated for the presence of PD signal, enthesophytes and calcifications. All the US lesions were scored using a dichotomous scale (presence/absence). All US exams were performed by an expert sonographer blind to clinical history, using an Esaote MyLab70 scanner equipped with high resolution linear probes.

Results: 11 patients ( 6 women) were enrolled in this study with a mean age of 57 yo $(S D \pm 11,50)$. the mean number of joints with effusion was 3,9 for each patient (median 3, range 2-8) while the mean number of joints with synovial hypertrophy was of 2,9 (median 2, range $2-7$ ). $=0,18$ joints (median 0 , range $0-2$ ) presented also PD signal. I The mean number of exudative tenosynovitis was 0,81 (median 2 , range $0-3$ ) while proliferative tenosynovitis (mean 0,54 , median 0 , range $0-2$ ) and PD in tendons with sheaths (mean 0,27, median 0, range 0-2) were rare. Finally, the mean number of enthesis with PD was 1,27 (median 1, range 0-7), the mean number of enthesophytes was 0,63 (median 0 , range $0-3$ ) and for calcifications 4,27 (median 5, range 1-8).

Conclusions: Ochronotic arthropathy is believed to be characterized by a widespread articular damage, correlated mainly to degenerative processes due to the deposition of Homogentisinic Acid in the joints. The results of this US study 